\title{
CONTINUITY AND DIFFERENTIABILITY PROPERTIES OF THE NEMITSKII OPERATOR IN HÖLDER SPACES
}

\author{
by RITA NUGARI
}

(Received 16 March, 1986)

Introduction. Let $\mathbb{R}^{n}$ be the $n$-dimensional Euclidean space with the usual norm denoted by $|\cdot|$. In what follows $\Omega$ will denote an open bounded subset of $\mathbb{R}^{n}$, and $\bar{\Omega}$ its closure.

For $\alpha \in(0,1], C^{0, \alpha}(\bar{\Omega}, \mathbb{R})$ is the space of all functions $u: \bar{\Omega} \rightarrow \mathbb{R}$ such that:

$$
h_{\alpha}(u):=\sup \left\{|u(x)-u(y)| /|x-y|^{\alpha} ; x, y \in \bar{\Omega}, x \neq y\right\}<\infty .
$$

$C^{0, \alpha}(\bar{\Omega}, \mathbb{R})$ is called the Hölder space with exponent $\alpha$ and is a Banach space when endowed with the norm:

$$
\|u\|_{0, \alpha}=\|u\|_{\infty}+h_{\alpha}(u),
$$

where $\|u\|_{\infty}$ is, as usual, defined by:

$$
\|u\|_{\infty}=\sup \{|u(x)| ; x \in \bar{\Omega}\} .
$$

Let moreover $f=f(x, t)$ be a real valued function defined on $\bar{\Omega} \times \mathbb{R}$.

The aim of this paper is to find conditions on $f$ ensuring some continuity and differentiability properties of the so called Nemitskii operator induced by $f$; i.e. the operator $F$ defined by

$$
F(u)(x)=f(x, u(x)) \quad(x \in \bar{\Omega})
$$

for real valued functions $u$ defined on $\bar{\Omega}$.

More precisely we show that:

(a) if $f$ satisfies the assumption

$$
f \in C^{0,1}(\overline{\mathbf{\Omega}} \times \bar{I}, \mathbb{R}) \text { for any bounded interval } I \subset \mathbb{R},
$$

then $F$ maps $C^{0, \alpha}(\bar{\Omega}, \mathbb{R})$ into itself;

(b) if $f=f(x, t)$ is differentiable with respect to the real variable $t$ and its derivative $f_{t}^{\prime}(x, t)$ satisfies $(\mathrm{H})$, then $F$ maps $C^{0, \alpha}(\bar{\Omega}, \mathbb{R})$ continuously into itself;

(c) finally, if $f$ is twice differentiable with respect to $t$ and the second derivative $f_{t}^{\prime \prime}$ satisfies $(\mathrm{H})$, then $F$ is continuously differentiable.

The same results can be obtained if $f$ is a real valued function defined on $\bar{\Omega} \times \mathbb{R}^{m}(m \geqq 1)$; the corresponding statements are given in $\S 3$.

Continuity properties of the Nemitskii operator operator in Sobolev spaces rather than in Hölder spaces are proved by Valent in [3]; he shows (Theorem 2) that if $\Omega$ has the cone property, if $f \in C^{m}(\bar{\Omega} \times \mathbb{R})$ and $m p>n$, then $F$ maps $W^{m, p}(\Omega)$ continuously into itself.

We end this note with an application of the results above in the degree-theoretical Glasgow Math. J. 30 (1988) 59-65. 
approach to non linear elliptic boundary value problems of the kind:

$$
\left\{\begin{aligned}
f\left(x, u, D u, D^{2} u\right) & =0 \quad(\text { in } \Omega) \\
u & =0 \quad(\text { on } \partial \Omega) .
\end{aligned}\right.
$$

1. Continuity. Let $\Omega$ and $f$ be as in the Introduction. In this section we state conditions on $f$ ensuring that the corresponding Nemitskii operator maps $C^{0, \alpha}(\bar{\Omega}, \mathbb{R})$ into itself and is continuous.

THEOREM 1.1. If $f$ satisfies $(H)$, then $F$ maps $C^{0, \alpha}(\bar{\Omega}, \mathbb{R})$ into itself.

Proof. Let $u \in C^{0, \alpha}(\bar{\Omega}, \mathbb{R})$ and $M=\|u\|_{0, \alpha}$; then $|u(x)| \leqq M \forall x \in \bar{\Omega}$. Let moreover $\bar{I}=[-M, M]$ and $k=k(I)$ be the Lipschitz constant of $f$ relative to $I$. Then

$$
|f(x, u(x))-f(y, u(y))| /|x-y|^{\alpha} \leqq k\left\{(|x-y|+|u(x)-u(y)|) /|x-y|^{\alpha}\right\} \quad(x, y \in \bar{\Omega}) .
$$

If $d$ denotes $(\operatorname{diam} \Omega)^{1-\alpha}$, one gets

$$
h_{\alpha}(F(u)) \leqq k\left\{d+h_{\alpha}(u)\right\} .
$$

Moreover, for any $(x, t)$ in $\bar{\Omega} \times \bar{I}$,

$$
|f(x, t)| \leqq c+k\left\{\left|x-x_{0}\right|+|t|\right\},
$$

where $x_{0}$ is an arbitrary point in $\bar{\Omega}$ and $c=\left|f\left(x_{0}, 0\right)\right|$.

Therefore, for all $x \in \bar{\Omega}$,

$$
|f(x, u(x))| \leqq c+k\left(c_{1}+\|u\|_{\infty}\right),
$$

where $c_{1}$ is the radius of a ball centered at $x_{0}$ and containing $\bar{\Omega}$.

Finally, taking into account (1.1) and (1.2), we get

$$
\|F(u)\|_{0, \alpha} \leqq c+k\left(c_{2}+\|u\|_{0, \alpha}\right),
$$

where $c_{2}=d+c_{1}$.

THEOREM 1.2. Let $f_{t}^{\prime}$ denote the partial derivative of $f$ with respect to the real variable $t$ and assume that $f_{1}^{\prime}$ satisfies $(H)$. Then:

(i) the Nemitskii operator $G$ induced by $f_{i}^{\prime}$ maps $C^{0, \alpha}(\bar{\Omega}, \mathbb{R})$ into itself;

(ii) the Nemitskii operator $F$ induced by $f$ is locally Lipschitzian and hence continuous.

Proof. (i) is a consequence of Theorem 1.1. (ii) Fix $u \in C^{0, \alpha}(\bar{\Omega}, \mathbb{P})$, let $N=$ $\|u\|_{0, \alpha}+1, \bar{J}=[-N, N]$ and let $k$ be the Lipschitz constant of $f_{t}^{\prime}$ corresponding to $\bar{J}$. Then, arguing as in the proof of Theorem 1.1, we get

$$
\|G(u+\xi v)\|_{0, \alpha} \leqq c+k\left(c_{2}+\|u+\xi v\|\right)
$$


whenever $\xi \in[0,1]$ and $v \in C^{0, \alpha}(\bar{\Omega}, \mathbb{R})$ is such that $\|v\|_{0, \alpha} \leqq 1$. Now write

$$
\begin{aligned}
f(x, u(x)+v(x))-f(x, u(x)) & =\int_{0}^{1} f_{i}^{\prime}(x, u(x)+\xi v(x)) v(x) d \xi \\
& =\int_{0}^{1} G(u+\xi v)(x) v(x) d \xi,
\end{aligned}
$$

whence

$$
\|F(u+v)-F(u)\|_{\infty} \leqq \int_{0}^{1}\|G(u+\xi v) v\|_{\infty} d \xi .
$$

From (1.4) we also get

$$
\begin{aligned}
&|f(x, u(x)+v(x))-f(x, u(x))-f(y, u(y)+v(y))+f(y, u(y))| /|x-y|^{\alpha} \\
& \leqq \int_{0}^{1}|G(u+\xi v)(x) v(x)-G(u+\xi v)(y) v(y)| /|x-y|^{\alpha} d \xi,
\end{aligned}
$$

which shows that

$$
h_{\alpha}(F(u+v)-F(u)) \leqq \int_{0}^{1} h_{\alpha}(G(u+\xi v) v) \mathrm{d} \xi
$$

Therefore, from (1.5) and (1.7),

$$
\|F(u+v)-F(u)\|_{0, \alpha} \leqq \int_{0}^{1}\|G(u+\xi v) v\|_{0, \alpha} d \xi .
$$

One checks easily that $\|w v\|_{0, \alpha} \leqq m\|w\|_{0, \alpha}\|v\|_{0, \alpha}$ for some $m \geqq 0$ and all $w, v \in$ $C^{0, \alpha}(\bar{\Omega}, \mathbb{R})$; therefore we have

$$
\|F(u+v)-F(u)\|_{0, \alpha} \leqq m\|v\|_{0, \alpha} \int_{0}^{1}\|G(u+\xi v)\|_{0, \alpha} d \xi
$$

whence, using (1.3), we finally get, if $\|v\|_{0, \alpha} \leqq 1$,

$$
\|F(u+v)-F(u)\|_{0, \alpha} \leqq L\|v\|_{0, \alpha},
$$

where $L=m\left[c+k\left(c_{2}+N\right)\right]$. This proves that $F$ is Lipschitz continuous around $u$.

\section{Differentiability}

THEOREM 2.1. Let $\Omega$ be as before, let $f$ be twice differentiable with respect to the real variable $t$, and assume that its second derivative $f_{t}^{\prime \prime}$ satisfies $(H)$. Then:

(i) the Nemitskii operator $G$ induced by $f_{t}^{\prime}$ is continuous;

(ii) the Nemitskii operator $F$ induced by $f$ is continuously differentiable, with derivative $F^{\prime}(u)[v]=G(u) v$. 
Proof. (i) This is a consequence of Theorem 1.2. (ii) Set

$$
w(u, v, x):=f(x, u(x)+v(x))-f(x, u(x))-f_{t}^{\prime}(x, u(x)) v(x)
$$

so that

$$
\begin{aligned}
w(u, v, x) & =\int_{0}^{1}\left[f_{t}^{\prime}(x, u(x)+\xi v(x))-f_{t}^{\prime}(x, u(x))\right] v(x) d \xi \\
& =\int_{0}^{1}(G(u+\xi v)-G(u))(x) v(x) d \xi
\end{aligned}
$$

whence

$$
\|F(u+v)-F(u)-G(u) v\|_{\infty} \leqq \int_{0}^{1}\|(G(u+\xi v)-G(u)) v\|_{\infty} d \xi
$$

Moreover,

$$
\begin{aligned}
\mid w(u, v, x)- & w(u, v, y)|/| x-\left.y\right|^{\alpha} \\
& \leqq \int_{0}^{1}|(G(u+\xi v)-G(u))(x) v(x)-(G(u+\xi v)-G(u))(y) v(y)| /|x-y|^{\alpha} d \xi .
\end{aligned}
$$

In other words,

$$
h_{\alpha}[F(u+v)-F(u)-G(u) v] \leqq \int_{0}^{1} h_{\alpha}[(G(u+\xi v)-G(u)) v] d \xi .
$$

We conclude that

$$
\begin{aligned}
\|F(u+v)-F(u)-G(u) v\|_{0, \alpha} \leqq \int_{0}^{1} \|(G(u+\xi v) & -G(u)) v \|_{0, \alpha} d \xi \\
& \leqq m\|v\|_{0, \alpha} \int_{0}^{1}\|G(u+\xi v)-G(u)\|_{0, \alpha} d \xi .
\end{aligned}
$$

Now let $\varepsilon>0$. By continuity of $G$ (part (i)) there exists $\delta>0$ such that $\|G(u+\xi v)-G(u)\|_{0, \alpha}<\varepsilon$ whenever $\|v\|_{0, \alpha}<\delta$. Therefore,

$$
\|F(u+v)-F(u)-G(u) v\|_{0, \alpha} \leqq \varepsilon\|v\|_{0, \alpha}
$$

whenever $\|v\|_{0, \alpha}<\delta$, showing that $F$ is differentiable at $u$ with derivative $F^{\prime}(u)[v]=$ $G(u) v$.

Finally, to show that $F$ is continuously differentiable, let $\mathscr{L}$ denote the Banach space of all linear bounded mappings of $C^{0, \alpha}(\bar{\Omega}, \mathbb{R})$ into itself, equipped with its usual norm $\|T\|_{\mathscr{L}}=\sup \left\{\|T[v]\|_{0, \alpha}:\|v\|_{0, \alpha}=1\right\}$.

Since

$$
\begin{aligned}
&\left\|F^{\prime}(u+w)[v]-F^{\prime}(u)[v]\right\|_{0, \alpha}=\|G(u+w) v-G(u) v\|_{0, \alpha} \\
& \leqq m\|G(u+w)-G(u)\|_{0, \alpha}\|v\|_{0, \alpha}
\end{aligned}
$$


we have

$$
\left\|F^{\prime}(u+w)-F^{\prime}(u)\right\|_{\mathscr{L}} \leqq m\|G(u+w)-G(u)\|_{0, \alpha}
$$

and the conclusion follows again from the continuity of $G$.

3. Vector-valued functions. If $\Omega$ denotes, as before, an open bounded subset of $\mathbb{R}^{n}$, the same results given in Sections 1 and 2 can be stated when $f=f(x, s)=$ $f\left(x, s_{1}, \ldots, s_{m}\right)$ is a real-valued function defined on $\bar{\Omega} \times \mathbb{B}^{m}(m \geqq 1)$.

We let here $f_{s}^{\prime}=\left(f_{s_{1}}^{\prime}, \ldots, f_{s_{m}}^{\prime}\right)$ denote the gradient of $f$ with respect to the variable $s \in \mathbb{R}^{m}$, while $f_{s}^{\prime \prime}$ will denote the $m \times m$ Hessian matrix $\left(f_{s_{s_{j}}}^{\prime \prime}\right)(i, j=1, \ldots, m)$ of $f$ with respect to the same variable.

Moreover, the symbol $I$ will denote here a bounded interval in $\mathbb{R}^{m}$ :

$$
I=\left\{x=\left(x_{1}, \ldots, x_{m}\right) \in \mathbb{R}^{m}: a_{i}<x_{i}<b_{i}, i=1,2, \ldots, m\right\}
$$

(with $a_{i}, b_{i}$ real numbers such that $a_{i}<b_{i}, i=1, \ldots, m$ ) and $\bar{I}$ will denote the closure of $\bar{I}$.

Finally, we choose for the space $C^{0, \alpha}\left(\bar{\Omega}, \mathbb{R}^{m}\right)$ the norm:

$$
\|u\|_{0, \alpha}=\sum_{i=1}^{m}\left\|u_{i}\right\|_{0, \alpha} \quad\left(u=\left(u_{1}, u_{2}, \ldots, u_{m}\right)\right) .
$$

THEOREM 3.1. Let $\Omega$ be as before and let $f: \bar{\Omega} \times \mathbb{R}^{m} \rightarrow \mathbb{R}$ be of class $C^{0,1}(\bar{\Omega} \times \bar{I}, \mathbb{R})$ for any bounded interval $I \subset \mathbb{R}^{m}$; then the Nemitskii operator $F$ induced by $f$, defined by $F(u)(x)=f(x, u(x))$ for vector valued functions $u: \bar{\Omega} \rightarrow \mathbb{R}^{m}$, maps $C^{0, \alpha}\left(\bar{\Omega}, \mathbb{R}^{m}\right)$ into $C^{0, \alpha}(\bar{\Omega}, \mathbb{R})$.

THEOREM 3.2. With the same notations as before, assume moreover that $f$ is differentiable with respect to the $\mathbb{R}^{m}$ variable and that $f_{s}^{\prime} \in C^{0,1}\left(\bar{\Omega} \times \bar{I}, \mathbb{R}^{m}\right)$ for any bounded interval $I \subset \mathbb{R}^{m}$. Then:

(i) the Nemitskii operator $G$ induced by $f_{s}^{\prime}$ maps $C^{0, \alpha}\left(\bar{\Omega}, \mathbb{R}^{m}\right)$ into itself;

(ii) the Nemitskii operator $F$ induced by $f$ maps $C^{0, \alpha}\left(\bar{\Omega}, \mathbb{R}^{m}\right)$ into $C^{0, \alpha}(\bar{\Omega}, \mathbb{R})$ and is locally Lipschitzian.

THEOREM 3.3. If $f$ is twice differentiable with respect to the $\mathbb{R}^{m}$ variable and $f_{s}^{\prime \prime} \in C^{0,1}\left(\bar{\Omega} \times \bar{I}, \mathbb{R}^{m}\right)$ for any bounded interval $I \subset \mathbb{R}^{m}$, then:

(i) the Nemitskii operator $G$ induced by $f_{t}^{\prime}$ maps continuously $C^{0, \alpha}\left(\bar{\Omega}, \mathbb{R}^{m}\right)$ into itself;

(ii) the Nemitskii operator $F$ induced by $f$ maps $C^{0, \alpha}\left(\bar{\Omega}, \mathbb{R}^{m}\right)$ into $C^{0, \alpha}(\bar{\Omega}, \mathbb{R})$ and is continuously differentiable with derivative

$$
\left(F^{\prime}(u)[v]=G^{\prime}(u) \cdot v \quad\left(u, v \in C^{0, \alpha}\left(\bar{\Omega}, \mathbb{R}^{m}\right)\right),\right.
$$

where $\cdot$ denotes the scalar product in $\mathbb{B}^{m}$; explicitly,

$$
\begin{aligned}
\left(F^{\prime}(u)[v]\right)(x) & =f_{s}^{\prime}(x, u(x)) \cdot v(x) \\
& =\sum_{i=1}^{m} f_{s_{i}}^{\prime}(x, u(x)) v_{i}(x) .
\end{aligned}
$$


4. An application to nonlinear elliptic problems. Let $C^{2, \alpha}(\bar{\Omega}, \mathbb{R})$ be the space of real functions defined on $\bar{\Omega}$, with derivative up to the second order in $C^{0, \alpha}(\bar{\Omega}, \mathbb{R})$. We equip $C^{2, \alpha}(\bar{\Omega}, \mathbb{R})$ with the usual norm:

$$
\|u\|_{2, \alpha}=\sum_{|k| \leqq 2}\left\|D^{k} u\right\|_{0, \alpha}
$$

where $k=\left(k_{1}, \ldots, k_{n}\right)$ is a multiindex, $|k|=k_{1}+\ldots+k_{n}$ and

$$
D^{k} u=\frac{\partial^{|k|} u}{\partial^{k_{1}} x_{1} \ldots \partial^{k_{n}} x_{n}} .
$$

Let moreover $f=f(x, t, p, q)$ be a real valued function defined on $\bar{\Omega} \times \mathbb{R} \times \mathbb{R}^{n} \times$ $\mathbb{R}^{n^{2}}=\bar{\Omega} \times \mathbb{R}^{m}\left(m=1+n+n^{2}\right)$, and consider the following nonlinear boundary value problem:

$$
\left\{\begin{aligned}
f\left(x, u, D u, D^{2} u\right) & =0 \quad(\text { in } \Omega), \\
u & =0 \quad(\text { on } \partial \Omega)
\end{aligned}\right.
$$

where $\Omega$ has smooth boundary $\partial \Omega$ and $D u, D^{2} u$ are shorthand notations for the first (resp. second) order derivatives of $u$.

One seeks $C^{2, \alpha}$ solutions of (4.1).

One way of attacking (4.1) is to use degree theory for Fredholm mappings, as suggested by K. D. Elworthy and A. J. Tromba in their paper [2]. To do this, one basic requirement to fulfill is that the Nemitskii operator $F$ induced by $f$ be a smooth (e.g. $C^{1}$ ) mapping between $C^{2, \alpha}(\bar{\Omega} ; \mathbb{R})$ and $C^{0, \alpha}(\bar{\Omega}, \mathbb{R})$; moreover, one needs the explicit expression of the derivative $F^{\prime}(u)$ in order to check that $F$ is a Fredholm mapping of index zero (see e.g. Berger [1] for the definition). To this end we prove the following result.

THEOREM 4.1. Let $f=f(x, t, p, q)$ be as above and assume that it satisfies the assumptions of Theorem 3.3. Then the induced Nemitskii operator

$$
\bar{F}(u)(x)=f\left(x, u(x), D u(x), D^{2} u(x)\right) \quad(x \in \bar{\Omega})
$$

maps $C^{2, \alpha}(\bar{\Omega}, \mathbb{R})$ into $C^{0, \alpha}(\bar{\Omega}, \mathbb{R})$ and is continuously differentiable, with derivative

$$
\begin{aligned}
& \left(\bar{F}^{\prime}(u)[v]\right)(x)=f_{t}^{\prime}\left(x, u(x), D u(x), D^{2} u(x)\right) v(x) \\
& +\sum_{i=1}^{n} f_{p_{i}}^{\prime}\left(x, u(x), D u(x), D^{2} u(x)\right) \frac{\partial v}{\partial x_{i}}(x) \\
& +\sum_{i, j=1}^{n} f_{q_{i, j}}^{\prime}\left(x, u(x), D u(x), D^{2} u(x)\right) \frac{\partial^{2} v}{\partial x_{i} \partial x_{j}}(x)
\end{aligned}
$$

for any $u, v \in C^{2, \alpha}(\bar{\Omega}, \mathbb{R})$.

Proof. Let $j$ be the isometry of $C^{2, \alpha}(\bar{\Omega}, \mathbb{R})$ onto $C^{0, \alpha}\left(\bar{\Omega}, \mathbb{R}^{m}\right)$, defined by

$$
j u=\left(u, D u, D^{2} u\right),
$$


and let $F$ be the Nemitskii operator induced by $f$ on $C^{0, \alpha}\left(\bar{\Omega}, \mathbb{R}^{m}\right)$; i.e.

$$
F(v)(x)=f(x, v(x)), \quad v \in C^{0, \alpha}\left(\bar{\Omega}, \mathbb{R}^{m}\right) .
$$

We have

$$
\bar{F}(u)=F(j u) \quad\left(v \in C^{2, \alpha}(\bar{\Omega}, \mathbb{R})\right) ;
$$

i.e. $\bar{F}=F \circ j$. Therefore, by Theorem (3.3), $\bar{F}$ maps continuously $C^{2, \alpha}(\bar{\Omega}, \mathbb{R})$ into $C^{0, \alpha}(\bar{\Omega}, \mathbb{R})$ and is continuously differentiable; moreover, by the chain rule,

$$
\bar{F}^{\prime}(u)=F^{\prime}(j u) \circ j
$$

or

$$
\bar{F}(u)[v]=F^{\prime}(j u)[j v] \quad\left(u, v \in C^{2, \alpha}(\bar{\Omega}, \mathbb{R})\right) .
$$

Therefore, by the explicit formula (3.1),

$$
\begin{aligned}
\left(\bar{F}^{\prime}(u)\right)[v](x) & =f_{s}^{\prime}(x, j u(x)) \cdot j v(x) \\
& =f_{s}^{\prime}\left(x, u, D u(x), D^{2} u(x)\right) \cdot\left(v(x), D v(x), D^{2} v(x)\right),
\end{aligned}
$$

which is nothing but the shorthand version of (4.2).

\section{REFERENCES}

1. M. S. Berger, Nonlinearity and functional analysis, (Academic Press, 1977).

2. K. D. Elworthy and A. J. Tromba, Degree theory on Banach manifolds, Proc. Symp. Pure Mathematics 18 (AMS, Providence, 1970).

3. T. Valent, A property of multiplication in Sobolev spaces. Some applications, Rend. Sem. Mat. Univ. Padova 74 (1985), 63-73.

Università dègli Studi della Calabria

Dipartimento di Matematica

87036 Arcavacata di Rende (Cosenza)

ITALY 